\title{
A novel simple experimental model for low-osmolar contrast-induced acute kidney injury using different definitions based on the levels of serum creatinine and cystatin $\mathrm{C}$
}

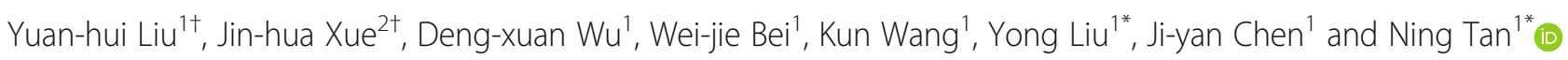

\begin{abstract}
Background: It remained lack of a kind of contrast-induced acute kidney injury (Cl-AKI) model which was widely used in clinical practice and comparable to Cl-AKI in humans.

Methods: Fifty Sprague-Dawley rats were divided into five groups of 10 rats each: (1) sham group (normal saline [NS] + NS); (2) NS plus low osmolality contrast medium (CM15) (NS + CM15); (3) furosemide (FM) plus NS (FM + NS); (4) FM + CM10; and (5) FM + CM15.We measured the levels of serum creatinine ( $\mathrm{SCr}$ ), cystatin C (cys-C) and histopathological scores of kidney tissues.

Results: $\mathrm{SCr}$ level in the FM + CM15 group were significantly increased after CM exposure compared with baseline levels ( $32.9 \pm 4.57$ vs. $158.7 \pm 14.48 \mu \mathrm{mol} / \mathrm{L}, p<0.001)$. Minor changes were found about the $\mathrm{SCr}$ levels between the preand post-exposure CM or NS treatment in the other groups. Additionally, the cys-C levels after CM exposure were increased compared with pretreatment levels in the FM+CM15 group ( $0.08 \pm 0.03$ vs. $0.18 \pm 0.05 \mathrm{mg} / \mathrm{L}, p<0.001)$. Minor changes were noted in the FM + NS group before and after NS administration. Only rats in the FM + CM15 group developed $\mathrm{Cl}$-AKI with the definitions of $\mathrm{SCr}$ or cys-C. Comparing to the FM + NS group, the histopathological scores were significantly increased in the FM + CM15 group.
\end{abstract}

Conclusions: A simple and reliable animal model for low osmolality contrast medium-induced AKI was established, which is similar to clinical Cl-AKI based on different definitions for AKI.

Keywords: Contrast, Acute kidney injury, Model

\section{Background}

Contrast-induced acute kidney injury (CI-AKI) is a serious common complication in patients after contrast medium (CM) exposure during cardiac catheterization and computed tomography. CI-AKI increases the length of hospital stay, health care cost, and rate of in-hospital

\footnotetext{
*Correspondence: liuyong2099@126.com; gdtanning@126.com

${ }^{\dagger}$ Yuan-hui Liu and Jin-hua Xue contributed equally to this work.

'Department of Cardiology, Guangdong Cardiovascular Institute, Guangdong Provincial Key Laboratory of Coronary Heart Disease Prevention, Guangdong Provincial People's Hospital, Guangdong Academy of Medical Sciences, Guangzhou 510100, Guangdong, China

Full list of author information is available at the end of the article
}

complications, including a mortality rate of approximately $20 \%$, and patients may become predisposed to long-term loss of kidney function [1-4]. However, other than preprocedural hydration and limiting the CM volume, few strategies have been proven effective for preventing CI-AKI [5]. Although an extensive amount of work has been performed during the past 20 years, the pathophysiology of CI-AKI remains obscure [6]. The mechanisms involved in CI-AKI play important roles in understanding and preventing CI-AKI. Nevertheless, progress in understanding this pathology seems to have been hampered by the lack of a reliable and reproducible experimental model for CI-AKI. 
Unfortunately, most previous CI-AKI models are not appropriate given that they were induced in rats by the intravenous injection of a high osmolality CM, in addition to reagents that inhibit prostaglandin (indomethacin) and nitric oxide synthesis [7-9], which are associated with the greatest risk of developing CI-AKI and are not used in clinical practice. In contrast, a low or iso-osmolality CM is widely used in clinical settings. Nevertheless, few studies have established an experimental model for CI-AKI based on low osmolality CM (LOCM) without other nephrotoxic drugs, that would be comparable to clinical CI-AKI in humans. In addition, more novel biomarkers for detecting CI-AKI have been reported. Cystatin $\mathrm{C}$ (cys-C) provides increased sensitivity with equivalent specificity for detecting CI-AKI compared to serum creatinine ( $\mathrm{SCr}$ ). Therefore, the present study aimed to develop an experimental model for LOCM-induced AKI with different definitions for AKI based on $\mathrm{SCr}$ and cys-C to develop new mechanisms or strategies for preventing CI-AKI in the future.

\section{Methods}

\section{Ethic statements}

The experimental procedures were finished by the guiding of the Care and Use of Laboratory Animals, which was published by the US National Institutes of Health And it was also approved by the Institutional Review Board of the Blinded and Hospital, Guangdong, China. Adult male Sprague-Dawley rats were purchased from the Laboratory Animal Science Department of the Blinded (License No. SYXK (Yue) 2010-0056).

\section{Animals}

We used adult male Sprague-Dawley rats aged 8-10 weeks old (190-230 g). The rats were maintained in standard housing facilities with a $12 \mathrm{~h}$ light $/ 2 \mathrm{~h}$ dark cycle at $24 \pm 1{ }^{\circ} \mathrm{C}$ and $45 \pm 5 \%$ humidity. At the beginning of the study, the rats were acclimatized for 7 days. The experimental procedures were performed by the guiding of our institutional and national guidelines for animal research.

\section{Experimental design and the contrast medium}

The rats were randomly allocated into the following five groups ( $n=10$ for each group and each experiment): (1) sham (normal saline [NS] + NS), (2) NS plus contrast medium (CM15) (NS + CM15), (3) furosemide (FM) plus NS (FM + NS), (4) FM + CM10, and (5) FM + CM15.

After acclimatization for 7 days, and $6 \mathrm{~h}$ before CM administration, groups $\mathrm{FM}+\mathrm{NS}, \mathrm{FM}+\mathrm{CM}_{10}$ and $\mathrm{FM}+$ $\mathrm{CM}_{15}$ received furosemide (Harvest Pharmaceutical Co., GuangZhou, China), which was injected intramuscularly $(10 \mathrm{~mL} / \mathrm{kg})$. The other groups received the same volume of saline. Then, all the groups had restricted access to water for $6 \mathrm{~h}$. After $6 \mathrm{~h}$, groups $\mathrm{NS}+\mathrm{CM}_{15}$ and $\mathrm{FM}+\mathrm{CM}_{15}$ groups received LOCM (350 mg I/mL, Bayer, Guangzhou, China) in the tail vein at a dose of $15 \mathrm{~mL} / \mathrm{kg}$, whereas the $\mathrm{FM}+\mathrm{CM}_{10}$ group received $10 \mathrm{~mL} / \mathrm{kg}$ of $\mathrm{LOCM}$ for under ether anesthesia. The NS + NS, and FM + NS groups received the same dose of normal saline. Subsequently, all the rats were given unlimited access to standard rat chow and water. The rats were weighed before the furosemide and LOCM injections and at $24 \mathrm{~h}$ after CM administration. After $24 \mathrm{~h}$ following CM administration, rat blood was sampled to determine the SCr levels, and rats were sacrificed by decapitation. Their kidneys were removed for histological analyses.

\section{Renal function parameters}

Approximately $1.3 \mathrm{~mL}$ of blood was collected from the tail vein and was placed into a plain tube before LOCM or normal saline was injected under ether anesthesia. The blood was to be cloted at least $45 \mathrm{~min}$. Serum was collected after centrifugation at $2000 \times \mathrm{g}$ for $10 \mathrm{~min}$ and was analyzed for the $\mathrm{SCr}$ and cys-C levels. The final blood sample was analyzed at the end of the study (24 h) in the same manner. The $\mathrm{SCr}$ and cys- $\mathrm{C}$ measurements were performed using a biochemical automatic analyzer.

\section{Histological evaluation of kidneys}

Histological evaluation of kidneys was performed in accordance with previous researches $[10,11]$. The kidneys were excised and cut from the top to the bottom after $24 \mathrm{~h}$. Tissues were fixed in $10 \%$ neutral-buffered formalin and dehydrated in a graded series of alcohols. Then, tissues were deparaffinized in xylenes and were paraffinembedded. Subsequently, $5-\mu \mathrm{m}$ thick sections were cut from the paraffin blocks and were routinely dewaxed and hydrated. The slices were stained with hematoxylin, rinsed with water, differentiated with $1 \%$ hydrochloric acid alcohol, stained with eosin for $1 \mathrm{~min}$ and rinsed again with water. Finally, the slices were dehydrated with alcohol, deparaffinized in xylene again and mounted with cover slips. We performed the histopathological evaluation of the kidney glomeruli, tubules, interstitium, and arteries through a board-certified veterinary pathologist. And the process was blinded to the experimental groups. The extent of injury was based on the following criteria: no injury; mild; moderate; severe; and very severe $(0 ;<25 \%$; <50\%; $<75 \%$; $>75 \%$, respectively) [12].

\section{Definitions of $\mathrm{Cl}-\mathrm{AKI}$}

The CI-AKI was defined according to one of the following definitions: an absolute increase in SCr levels of $\geq 44.2 \mu \mathrm{mol} / \mathrm{L}$ or a relative increase of $\geq 25 \%$ from baseline within 48-72 h after CM exposure [13]; and an increase in the cys-C concentration of $10 \%$ greater than the baseline value at $24 \mathrm{~h}$ after the administration of CM [14]. 


\section{Statistical analysis}

Continuous variables are expressed as the mean \pm standard deviation or as their median (inter quartile range). Student's t-test or one-way analysis of variance was performed to determine differences among the groups. Categorical variables are reported as absolute values and percentages and they were analyzed using the chi-square test or Fisher's exact test. SAS version 9.2 (SAS Institute, Cary, NC, USA) was used to analysis. All the probability values were two-tailed and the statistical significance was defined as $p<0.05$.

\section{Results}

\section{Body weights}

The body weight was significantly reduced in all three groups $(\mathrm{FM}+\mathrm{NS}, \mathrm{FM}+\mathrm{CM} 10$, and $\mathrm{FM}+\mathrm{CM} 15)$ that were administered FM for dehydration. A limited number of changes were noted in the other groups that received NS. In addition, at the end of the experiment, the rat's body weights in the groups that were given $\mathrm{CM}$ and $\mathrm{FM}$ were significantly reduced compared with the other groups.

\section{$\mathrm{SCr}$ and cys- $\mathrm{C}$ concentrations}

The concentrations of $\mathrm{SCr}$ in each group before and after $\mathrm{CM}$ or NS exposure are presented in Table 1 . The $\mathrm{SCr}$ levels in the NS + NS, NS + CM15, FM + NS and $\mathrm{FM}+\mathrm{CM} 10$ groups were minimally changed between the pre- and post CM or NS exposure. However, in the $\mathrm{FM}+\mathrm{CM} 15$ group, the $\mathrm{SCr}$ concentration was significantly increased after CM exposure compared with baseline levels $(32.9 \pm 4.57$ vs. $158.7 \pm 14.48 \mathrm{umol} / \mathrm{L}, p<0.001)$. In addition, to confirm the change in the SCr of the FM + CM15 group, we also measured the levels of cys-C in $\mathrm{FM}+\mathrm{CM} 15$ and FM + NS groups to demonstrate the effect of CM15 on renal function. The cys-C levels were also increased after $\mathrm{CM}$ exposure compared with baseline in the $\mathrm{FM}+\mathrm{CM} 15$ group $(0.08 \pm 0.03$ vs. $0.18 \pm 0.05 \mathrm{mg} / \mathrm{L}$, $p<0.001)$. However, no significant difference was noted in the FM+NS, NS + NS and NS + CM15 group before and after NS administration $(0.07 \pm 0.01$ vs. $0.06 \pm$ $0.01 \mathrm{mg} / \mathrm{L} ; 0.03 \pm 0.01$ vs. $0.04 \pm 0.01 \mathrm{mg} / \mathrm{L} ; 0.04 \pm 0.01$ vs. $0.04 \pm 0.01 \mathrm{mg} / \mathrm{L}$; all $p>0.05)$.
Incidence of $\mathrm{Cl}-\mathrm{AKI}$ based on the $\mathrm{SCr}$ and Cys-c

CI-AKI was not induced based on the definition of $\mathrm{SCr}$ or cys-C in the NS+NS, NS + CM15, FM + NS and $\mathrm{FM}+\mathrm{CM} 10$ groups. Nevertheless, based on the definitions for $\mathrm{SCr}$ and cys-C, CI-AKI developed in the FM+ CM15 group.

\section{Histopathological results}

The histopathological scores of the FM + NS and FM + CM15 groups are presented in Fig. 1. Glomerular sclerosis and interstitial fibrosis were not observed in any of the groups. Noticeable detachment or foamy degeneration of tubular cells was not observed in the FM + NS group (histologic scoring: $0.40 \pm 0.52$; Fig. 1d-f). However, there was severe detachment and foamy degeneration of the tubular cells was noted in the FM + CM15 group (Fig. 1a-c), and the histologic scores were significantly increased compared with the $\mathrm{FM}+\mathrm{NS}$ group $(3.3 \pm 0.82, p<0.001)$.

\section{Discussion}

The present study established a novel simple CI-AKI model based on LOCM and different definitions of CIAKI, which included a novel biomarker for CI-AKI.

CI-AKI remains a serious complication in patients exposed to CM, and it is strongly associated with poor shortand long- term outcomes. Clinically relevant animal models of CI-AKI are useful for further understanding the mechanisms involved, identifying novel biomarkers, evaluating potential differences among different $\mathrm{CM}$ types, and identifying new strategies for preventing CI-AKI.

However, most of the previous CI-AKI animal models involved rats, and used indomethacin $(10 \mathrm{mg} / \mathrm{kg}$; intravenously, [IV]), followed by Nw-nitro- L-arginine methyl ester (L-NAME, $10 \mathrm{mg} / \mathrm{kg}$, IV) after $15 \mathrm{~min}$, and by $6 \mathrm{~mL} / \mathrm{kg}$ of high-osmolar radiological contrast agent $60 \%$ meglumine amidotrizoate injected into the tail vein after additional 15 min $[8,15,16]$. Experimental observations demonstrated that the use of nonsteroidal anti-inflammatory drugs (indomethacin) increased the risk of developing CI-AKI [17], and previous guidelines suggested that patients should be withdrawn from nephrotoxic drugs at least $24 \mathrm{~h}$ before $\mathrm{CM}$ administration [13]. In addition, contrast nephrotoxicity

Table 1 Changes of serum creatinine and body weight in each group

\begin{tabular}{|c|c|c|c|c|c|c|c|c|}
\hline \multirow{2}{*}{$\begin{array}{l}\text { Parameters } \\
\text { Times }\end{array}$} & \multicolumn{2}{|l|}{$\mathrm{SCr}(\mathrm{umol} / \mathrm{L})$} & \multirow{2}{*}{$\begin{array}{l}\text { Changes } \\
\text { Post-Pre }\end{array}$} & \multicolumn{4}{|c|}{ Body weight (mg) } & \multirow[b]{2}{*}{$24 h-6 h$} \\
\hline & Pre CM or NS & Post CM or NS & & Baseline & $6 \mathrm{~h}$ after FM or NS & 6 h-baseline & $24 \mathrm{~h}$ after $\mathrm{CM}$ or NS & \\
\hline $\mathrm{NS}+\mathrm{NS}$ & $17.6 \pm 2.07$ & $20.5 \pm 2.64$ & $1.1 \pm 1.5$ & $207.9 \pm 6.30$ & $206.8 \pm 6.18$ & $-3.0 \pm 2.6$ & $204.5 \pm 7.72$ & $-1.7 \pm 3.2$ \\
\hline $\mathrm{NS}+\mathrm{CM} 15$ & $17.6 \pm 2.07$ & $18.7 \pm 1.57$ & $2.9 \pm 2.7$ & $207.3 \pm 9.54$ & $204.20 \pm 9.09$ & $-1.2 \pm 4.2$ & $202.54 \pm 7.94$ & $-2.3 \pm 6.2$ \\
\hline $\mathrm{FM}+\mathrm{NS}$ & $36.2 \pm 6.27$ & $23.2 \pm 2.78$ & $-13.0 \pm 4.3$ & $203.80 \pm 6.1$ & $185.2 \pm 6.0$ & $-19.0 \pm 3.0$ & $198.03 \pm 15.23$ & $0.4 \pm 10.6$ \\
\hline $\mathrm{FM}+\mathrm{CM} 10$ & $38.6 \pm 6.85$ & $27.1 \pm 7.19$ & $-11.5 \pm 8.5$ & $203.9 \pm 9.60$ & $184.90 \pm 8.70$ & $-20.4 \pm 1.8$ & $185.30 \pm 8.88$ & $-8.7 \pm 11.1$ \\
\hline $\mathrm{FM}+\mathrm{CM} 15$ & $32.9 \pm 4.57$ & $158.7 \pm 14.48$ & $125.8 \pm 17.0$ & $202.6 \pm 8.20$ & $182.2 \pm 7.30$ & $-18.6 \pm 4.3$ & $173.50 \pm 12.62$ & $12.8 \pm 10.6$ \\
\hline
\end{tabular}

Abbreviation: NS normal saline, CM contrast medium, FM furosemide 

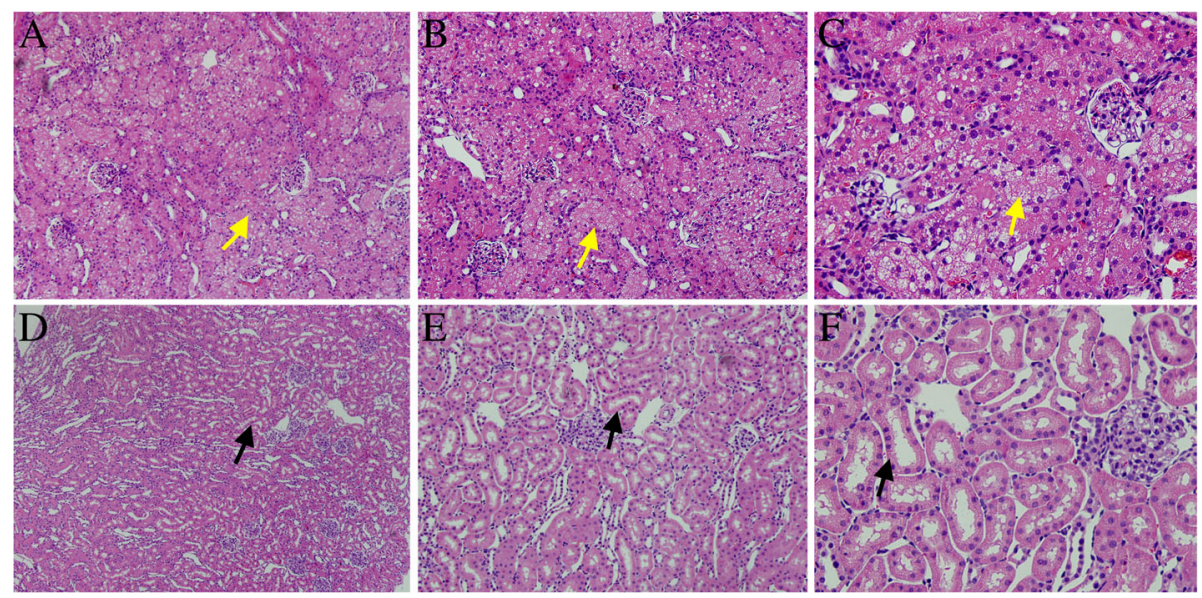

Fig. 1 Representative photomicrographs of the tubular cell injury in rat kidney tissue sections from the furosemide (FM) + normal saline and FM + contrast medium 15 groups. Original magnifications: $\times 100(\mathbf{a}$ and $\mathbf{d}), \times 200(\mathbf{b}$ and $\mathbf{e}$ ), and $\times 400$ (c and $\mathbf{f}$ ). Hematoxylin and eosin staining. Calibration bar $=20 \mu \mathrm{m}$. ( 7 :the lumen and structure of renal tubular was normal. The lumen of renal tubular was changed into small, even occlusion. And there is severe detachment and foamy degeneration of the tubular cells

and the risk of CI-AKI are related to the osmolarity of the $\mathrm{CM}$. It is widely accepted that a high osmolality $\mathrm{CM}$ is associated with the greatest risk of developing CI-AKI and is not used in clinical practice in patients who have been exposed to CM [18]. In contrast, the LOCMs were developed and are now widely used. Nevertheless, few animal models have systematically studied LOCM-induced AKI. The present study was performed to demonstrate this issue.

In clinical practice, the incidence of CI-AKI is very low in patients without any risk factors. In contrast, the CI-AKI incidence is significantly increased by up to $50 \%$ in patients presenting with multiple cardiovascular risk factors [19]. Therefore, CI-AKI cannot easily be induced by simply exposing healthy animals to $\mathrm{CM}$ alone, especially LOCM. Pretreating animals with factors similar to those considered to be risk factors for kidney damage in humans would be helpful for inducing CI-AKI. A previous study demonstrated that the manifestation of CMinduced renal vasoconstriction and oxidative stress, which are the important contributors of CI-AKI development, are the most prominent in a dehydrated animal. Therefore, numerous studies have considered water deprivation for $2-5$ days before $\mathrm{CM}$ exposure as a routine measure for inducing experimental CI-AKI. Instead of using water deprivation, we used FM before CM exposure to increase the urine output to achieve the same dehydration effect to some extent. We demonstrated that rats pretreated with FM at $6 \mathrm{~h}$ before CM exhibited decreased weight and an increased SCr levels, which would make them more prone to developing CI-AKI. Our findings demonstrated that FM with $\mathrm{CM}$ induces the development of CI-AKI. Additionally, the hematoxylin and eosin analysis indicated that the tubular cell injury in this group was more severe compared with controls. Studies by
Wang et al. [20] and Buyuklu et al. [21] used a CI-AKI model based on LOCM; however, they also used nephrotoxic drugs. Recently, Sun et al. [22] introduced the CIAKI model, which consisted of dehydration, FM, and Omnipaque. However, they only compared the FM + CM group with the $\mathrm{CM}$ group and they demonstrated that the levels of $\mathrm{SCr}$ in the former group reach the definitions of CI-AKI. Our findings were similar in that only CM was administered, which cannot induce CIAKI. However, we found that rats pre-treated with FM also have increased the SCr levels. Therefore, the study by Sun et al. should mimic our present study by adding another group $(\mathrm{FM}+\mathrm{NS})$ in order to exclude the effect of FM on increasing SCr levels.

In addition, most of the previous studies established the CI-AKI model according to the definition of CI-AKI as an increase in $\mathrm{SCr}$ of $\geq 25 \%$ of the baseline value within 48 $72 \mathrm{~h}$ [22, 23], which is not often used in clinical practice. The guidelines recommend the following definitions of CIAKI: an absolute increase in the SCr level of $\geq 44.2 \mu \mathrm{mol} / \mathrm{L}$ or a relative increase of $\geq 25 \%$ from baseline within $48-72$ $\mathrm{h}$ after $\mathrm{CM}$ exposure. Furthermore, clinical studies demonstrated that an increase in the SCr level of $\geq 44.2 \mu \mathrm{mol} / \mathrm{L}$ is more sensitive for selectively recognizing more patients with an increased risk of mortality and morbidity. However, $\mathrm{SCr}$ increases of $\geq 25 \%$ overestimate CI-AKI by including many patients without the postprocedural relevant deterioration of renal function, and they are affected by a reduced risk of adverse events at follow-up [24, 25]. Therefore, the present study established a CI-AKI model using these two definitions, which are closer to those used in clinical practice.

Although the definition of CI-AKI was based on the $\mathrm{SCr}$ concentration, $\mathrm{SCr}$ are affected by many status such 
as muscle catabolism. In addition, its rate of change after the initial insult is low. Moreover, the delayed increase in $\mathrm{SCr}$ is a potential reason for overlooking CI-AKI [26] or prolonging the hospital stay in the vast majority of patients who will not develop CI-AKI. However, cys-C is more sensitive than $\mathrm{SCr}$ for rapidly detecting acute changes in renal function and achieving a maximum within $24 \mathrm{~h}$ after $\mathrm{CM}$ exposure [27]. A previous study demonstrated that cys- $\mathrm{C}$ seems to be a reliable marker for the early diagnosis and prognosis of CI-AKI [14]. To the best of our knowledge, the current study may be the first to verify the experimental CI-AKI model based on cys-C.

\section{Limitations}

There were several limitations to the present study. Firstly, the dose of the CM was relatively high. However, it was not greater than the maximum safe contrast dose evaluated in a formula by Cigarroa et al. [28] in the humans. Second, we did not obtain the histopathological data or SCr levels at the different time points in order to better understand the development of CI-AKI. Third, we should note that there was a tipping point for AKI in the $\mathrm{CM}+\mathrm{FM}$ group, which can potentially be detected by various combinations of lower doses of FM and CM. Finally, we did not further investigate the mechanism of CI-AKI mainly through the inflammatory, apoptosis and ischemic/reperfusion.

\section{Conclusions}

In conclusion, a simple and reliable animal model for LOCM-induced AKI was developed for the first time. This model is similar to clinical CI-AKI based on different definitions for AKI.

\section{Abbreviations \\ Cl-AKI: Contrast-induced acute kidney injury; CM: Contrast medium; cys- C: cystatin C; FM: Furosemide; LOCM: Low osmolality CM; NS: Normal saline; SCr: Serum creatinine}

\section{Acknowledgements}

We acknowledged the help of Sheng-qi Wang, from the department of Mammary Disease, Guangdong Provincial Hospital of Chinese Medicine, The Second Clinical College of Guangzhou University of Chinese Medicine, Guangzhou, China.

The abstract of the manuscript was presented in The 28th Great Wall International Congress of Cardiology.

\section{Authors' contributions}

Conceived and designed the experiments: NT JYC LY. Performed the experiments: YHL WJB KW JHX DXW. Analyzed the data: YHL WJB KW JHX DXW. Contributed to the writing of the manuscript: YHL JHX YL. Contributed to revising manuscript critically for important intellectual content: NT JYC YL. And all authors have read and approve of the final version.

\section{Funding}

This study was supported by grant from the National Science Foundation for Young Scientists of China (grant no. 81800325), and National Natural Science Foundation of China (grant no. 81270286), Science and Technology Planning Project of Guangzhou City (grant no. 201906010089). The funders had no role in the study design, data collection and analysis, the decision to publish, or the preparation of the manuscript. The work was not funded by any industry sponsors.

\section{Availability of data and materials}

N/A.

Ethics approval and consent to participate

Guangdong Provincial People's Hospital ethics committee provided ethics approval. And the consent to participate was not applicable because of the basic research

\section{Consent for publication}

Not applicable.

\section{Competing interests}

The authors declare that they have no competing interests.

\section{Author details}

'Department of Cardiology, Guangdong Cardiovascular Institute, Guangdong Provincial Key Laboratory of Coronary Heart Disease Prevention, Guangdong Provincial People's Hospital, Guangdong Academy of Medical Sciences,

Guangzhou 510100, Guangdong, China. ${ }^{2}$ Department of Physiology, School of Basic Medical Sciences, Gannan Medical University, Ganzhou 341000,

China.

Received: 6 February 2018 Accepted: 24 June 2019

Published online: 04 July 2019

\section{References}

1. Tsai TT, Patel UD, Chang TI, et al. Contemporary incidence, predictors, and outcomes of acute kidney injury in patients undergoing percutaneous coronary interventions: insights from the NCDR Cath-PCI registry. JACC Cardiovasc Interv. 2014;7(1):1-9.

2. Wi J, Ko YG, Kim JS, et al. Impact of contrast-induced acute kidney injury with transient or persistent renal dysfunction on long-term outcomes of patients with acute myocardial infarction undergoing percutaneous coronary intervention. Heart. 2011;97(21):1753-7.

3. Lee J, Cho JY, Lee HJ, et al. Contrast-induced nephropathy in patients undergoing intravenous contrast-enhanced computed tomography in Korea:a multi-institutional study in 101487 patients. Korean J Radiol. 2014; 15(4):456.

4. Moos SI, van Vemde DNH, Stoker J, Bipat S. Contrast induced nephropathy in patients undergoing intravenous (IV) contrast enhanced computed tomography (CECT) and the relationship with risk factors: a meta-analysis. Eur J Radiol. 2013;82(9):e387-99.

5. van der Molen AJ, Reimer P, Dekkers IA, et al. Post-contrast acute kidney injury. Part 2: risk stratification, role of hydration and other prophylactic measures, patients taking metformin and chronic dialysis patients: Recommendations for updated ESUR Contrast Medium Safety Committee guidelines. Eur Radiol. 2018;28(7):2856-2869.

6. Seeliger E, Sendeski M, Rihal CS, Persson PB. Contrast-induced kidney injury: mechanisms, risk factors, and prevention. Eur Heart J. 2012;33(16):2007-15.

7. Ulusoy S, Ozkan G, Mungan S, et al. GSPE is superior to NAC in the prevention of contrast-induced nephropathy: might this superiority be related to caspase 1 and calpain 1? Life Sci. 2014;103(2):101-10.

8. Ari $E$, Kedrah AE, Alahdab $Y$, et al. Antioxidant and renoprotective effects of paricalcitol on experimental contrast-induced nephropathy model. Br J Radiol. 2012;85(1016):1038-43.

9. Homma K, Yoshida T, Yamashita M, Hayashida K, Hayashi M, Hori S. Inhalation of hydrogen gas is beneficial for preventing contrast-induced acute kidney injury in rats. Nephron Exp Nephrol. 2015.

10. Liu Y, Liu B, Liu Y, et al. MicroRNA expression profile by next-generation sequencing in a novel rat model of contrast-induced acute kidney injury. Ann Transl Med. 2019;7(8):178.

11. Liu T, Fang Y, Liu S, et al. Limb ischemic preconditioning protects against contrast-induced acute kidney injury in rats via phosphorylation of GSK-3 3 Free Radic Biol Med. 2015;81:170-82

12. Racusen LC, Solez K. Nephrotoxic tubular and interstitial lesions: morphology and classification. Toxicol Pathol. 1986;14(1):45-57.

13. Stacul F, van der Molen AJ, Reimer $P$, et al. Contrast induced nephropathy: updated ESUR contrast media safety committee guidelines. Eur Radiol. 2011; 21(12):2527-41.

14. Briguori C, Visconti G, Rivera NV, et al. Cystatin C and contrast-induced acute kidney injury. Circulation. 2010;121(19):2117-22. 
15. Li LP, Lu J, Zhou Y, et al. Evaluation of intrarenal oxygenation in iodinated contrast-induced acute kidney injury-susceptible rats by blood oxygen leveldependent magnetic resonance imaging. Investig Radiol. 2014;49(6):403-10.

16. Sahin I, Ozkaynak B, Sar M, et al. Paricalcitol counteracts the increased contrast induced nephropathy caused by renin-angiotensin-aldosterone system blockade therapy in a rat model. Eur Rev Med Pharmacol Sci. 2014; 18(19):2895-902

17. Morcos SK. Prevention of contrast media-induced nephrotoxicity after angiographic procedures. J Vasc Interv Radiol. 2005;16(1):13-23.

18. Tehrani S, Laing C, Yellon DM, Hausenloy DJ. Contrast-induced acute kidney injury following PCl. Eur J Clin Investig. 2013;43(5):483-90.

19. McCullough PA, Adam A, Becker CR, et al. Risk prediction of contrastinduced nephropathy. Am J Cardiol. 2006;98(6):27-36.

20. Wang F, Zhang G, Zhou Y, et al. Magnolin protects against contrast-induced nephropathy in rats via Antioxidation and Antiapoptosis. Oxidative Med Cell Longev. 2014;2014:1-7.

21. Buyuklu M, Kandemir F, Ozkaraca M, et al. Benefical effects of lycopene against contrast medium-induced oxidative stress, inflammation, autophagy, and apoptosis in rat kidney. Hum Exp Toxicol. 2015;34(5):487-96.

22. Sun $\mathrm{S}$, Zhang $T$, Nie $P$, et al. A novel rat model of contrast-induced acute kidney injury. Int J Cardiol. 2014;172(1):e48-50.

23. Liu T, Luo W, Tan X, et al. A novel contrast-induced acute kidney injury mode based on the 5/6-nephrectomy rat and Nephrotoxicological evaluation of lohexol and lodixanol. Oxidative Med Cell Longev. 2014;2014:1-14.

24. Budano C, Levis M, D'Amico M, et al. Impact of contrast-induced acute kidney injury definition on clinical outcomes. Am Heart J. 2011;161(5):963-71.

25. Slocum NK, Grossman PM, Moscucci M, et al. The changing definition of contrast-induced nephropathy and its clinical implications: insights from the blue cross blue shield of Michigan cardiovascular consortium (BMC2). Am Heart J. 2012;163(5):829-34

26. Solomon R, Barrett B. Follow-up of patients with contrast-induced nephropathy. Kidney Int Suppl. 2006;(100):S46-50.

27. Dharnidharka VR, Kwon C, Stevens G. Serum cystatin C is superior to serum creatinine as a marker of kidney function: a meta-analysis. Am J Kidney Dis. 2002;40(2):221-6.

28. Cigarroa RG, Lange RA, Williams RH, Hillis LD. Dosing of contrast material to prevent contrast nephropathy in patients with renal disease. Am J Med. 1989;86(6 Pt 1):649-52.

\section{Publisher's Note}

Springer Nature remains neutral with regard to jurisdictional claims in published maps and institutional affiliations.

Ready to submit your research? Choose BMC and benefit from:

- fast, convenient online submission

- thorough peer review by experienced researchers in your field

- rapid publication on acceptance

- support for research data, including large and complex data types

- gold Open Access which fosters wider collaboration and increased citations

- maximum visibility for your research: over $100 \mathrm{M}$ website views per year

At BMC, research is always in progress.

Learn more biomedcentral.com/submissions 